\title{
Kombinationstherapie mit Checkpoint-Inhibitor
}

\author{
Zum Einsatz von Checkpoint-Inhibitoren beim Prostatakarzinom gibt es \\ erst wenige Daten. In einer Studie soll nun die Wirksamkeit des Check- \\ point-Inhibitors Pembrolizumab nach Vortherapien untersucht werden.
}

$\mathrm{F}$ ür diese offene Phase-Ib/II-Studie zur Kombinationstherapie mit Pembrolizumab (MK-3475) bei Patienten mit metastasiertem kastrationsresistentem Prostatakarzinom (mCRPC) [KEY-
NOTE-365; Studie AP 94/16 der AUO] werden Patienten gemäß der Vortherapie in drei Kohorten eingeteilt. In Kohorte A erhalten Patienten, die als Vortherapie mit Docetaxel oder einer anderen Che-

\begin{abstract}
Tab. 1: Teilnehmende Studienzentren
Ort Kontaktdaten

Braunschweig Prof. Dr. Peter Hammerer, Tel.: 0531/595-2492, p.hammerer@klinikum-braunschweig.de

Homburg Prof. Dr. Carsten-Henning Ohlmann, Tel.: 06841/16-24700, carsten.ohImann@uniklinikum-saarland.de

Köln Prof. Dr. Jan Herden, Tel.: 0221/478-82108, jan.herden@uk-koeln.de

München

Münster

Nürnberg

Ulm

\begin{tabular}{l|l} 
Dr. Friedem \\
ulm.de
\end{tabular}
\end{abstract}

\section{Tab. 2: Ein- und Ausschlusskriterien (Auswahl)}

\section{Ausschlusskriterien}

- vorherige monoklonale Antikörpertherapie innerhalb von 4 Wochen vor Behandlungsbeginn

- Teilnahme an einer klinischen Prüfung innerhalb von 4 Wochen vor Therapiebeginn

_ diagnostizierte Immunschwäche oder systemische Steroidtherapie oder andere Form der Immunsuppression innerhalb von 7 Tagen vor Behandlungsbeginn

_ vorherige Radiumtherapie

- Vorliegen einer aktiven Autoimmunerkrankung mit Notwendigkeit einer systemischen Behandlung innerhalb der letzten 2 Jahre

- Anamnese von (nicht infektiöser) Pneumonitis, die eine Steroidtherapie erforderte oder aktive Pneumonitis

_ vorherige Teilnahme an anderen Pembrolizumab-Studien oder vorherige Therapie mit Anti-PD-1-, Anti-PD-L1-, Anti-PD-L2Präparat motherapie sowie mit bis zu zwei endokrinen Therapien der zweiten Generation, z.B. Abirateron, Enzalutamid, behandelt wurden, $200 \mathrm{mg}$ Pembrolizumab i.v. alle drei Wochen und 400 mg Olaparib zweimal täglich. Patienten der Kohorte B (Vortherapie Abirateron oder Enzalutamid) werden mit $200 \mathrm{mg}$ Pembrolizumab i.v. alle drei Wochen und $75 \mathrm{mg} /$ $\mathrm{m}^{2}$ Docetaxel i.v. alle drei Wochen (max. 10 Zyklen) behandelt. Patienten der Kohorte $\mathrm{C}$ (Vortherapie Abirateron und keine Chemotherapie; Vorbehandlung mit Docetaxel im hormonsensitiven Setting zulässig, so sie mind. vier Wochen zurückliegt) werden $200 \mathrm{mg}$ Pembrolizumab i.v. alle drei Wochen und $160 \mathrm{mg}$ Enzalutamid täglich verabreicht. Alle $\mathrm{Pa}$ tienten erhalten maximal 35 Zyklen (2 Jahre) Pembrolizumab, sofern kein Abbruch erforderlich oder das Therapieende gemäß Studienprotokoll erreicht wird.

Primäre Ziele sind Sicherheit und Verträglichkeit der Kombinationstherapien sowie die PSA-Ansprechraten. Sekundäre Ziele sind: Zeit bis PSA-Progression, radiologisch progressionsfreies Überleben, Gesamtüberleben, objektive Responserate, Dauer des Ansprechens und Krankheitskontrollrate.

In die Multizenterstudie sollen $210 \mathrm{~Pa}$ tienten (70 je Kohorte) eingebracht werden, davon circa 30 in sieben deutschen Zentren (Tab. 1). Tab. 2 enthält eine Auswahl der Ein- und Ausschlusskriterien.

Prof. Dr. med. Christian Wülfing Organgruppe Prostatakarzinom der AUO, Kuno-Fischer-Str. 8, 14057 Berlin

Prof. Dr. med. Margitta Retz

Leiterin der klinischen Prüfung, Klinikum r. d. Isar, Ismaninger Str. 22, 81675 München

\section{Heidrun Rexer}

MeckEvidence, Seestr. 11, 17252 Schwarz

E-Mail:AUO@MeckEvidence.de 